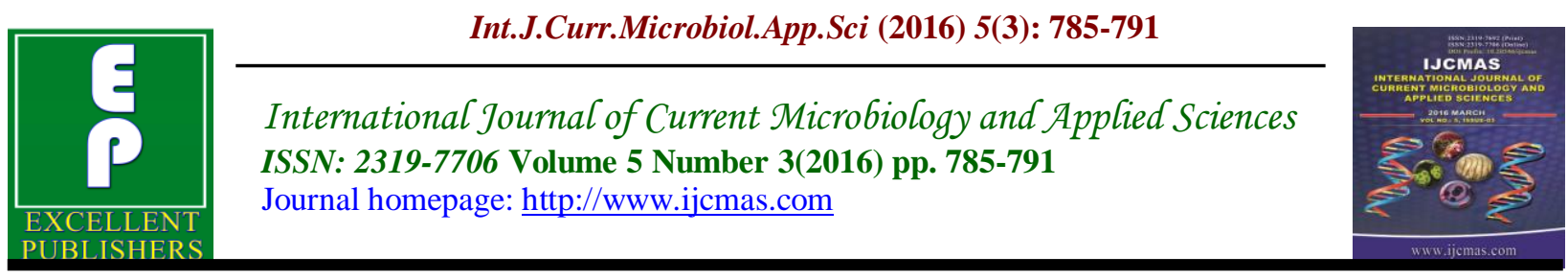

Original Research Article

http://dx.doi.org/10.20546/ijcmas.2016.503.091

\title{
Synthesis, Characterization and Antimicrobial Screening of Novel Ortho Hydroxy Chalcones
}

\author{
Abdulkareem Ali Hussien ${ }^{1,2 *}$, Bushra Ahmed Kateb ${ }^{1,2}$, \\ Shekh Saddam Husen ${ }^{3}$ and P. A. Kulkarni ${ }^{1}$ \\ ${ }^{1}$ Department of Chemistry, Yeshwant Mahavidyalaya, Nanded-431602 (MS), India \\ ${ }^{2}$ Hodiedah University, Education College, Yemen \\ ${ }^{3}$ Swami Ramanand Teerth University, Nanded, India \\ *Corresponding author
}

\section{A B S T R A C T}

Keywords

Chalcones,

Claisen-Schmidt condensation, Characterization, Antimicrobial activities.

\section{Article Info}

Accepted:

22 February 2016

Available Online:

10, March 2016
New series of chalcones(1a-j) were synthesized by Claisen-Schmidt condensation of 2-hydroxy, 5-methyl acetophenone with several aromatic aldehydes in presence of aqueous solution of sodium hydroxide.The synthesized chalcones compounds were characterized by Physical and spectral methods such as melting point, IR, ${ }^{1} \mathrm{H}-\mathrm{NMR}$ and Mass analysis. All the synthesized compounds have been screened and evaluated for antibacterial activity against Staphylococcus aureus gr +ve, Escherichia coli gr -ve Bacillus subtilis gr +ve, Salmonella typhi gr -ve, and antifungal activity against Aspergillus oryzoe, Aspergillus niger. DMSO was used as solvent control for their antimicrobial activity using disc diffusion method. Synthesis and biological evaluation of chalcones have been a topic of special interest to organic and medicinal chemists. The new structural classes of compounds may prove as lead molecules and good candidates for the future investigations.

\section{Introduction}

The chalcones (1, 3-diaryl-2-propenones) and their derivatives are important intermediates in organic synthesis (Straub, 1995; Sandler and Karo, 1972; Bergman Bergman et al., 1959). They serve as starting material for the synthesis of variety of heterocyclic compounds which are of physiological importance. Due to the presence of enone functionality in chalcone moiety confers biological activity upon it, like anti inflammatory anti-bacterial (Chikhalia et al., 2008), anti- cancer (Kotra et al., 2010), cytotoxic activity (Go et al., 2005), anti-hyperglycemic (Satyanarayana $e t$ al., 2004), (Ballesteros et al., 1995), antifungal, (Go et al., 2005), antioxidant (Mukerjee Mukerjee et al., 2001), antimalarial (Liu et al., 2003), antituberculosis (Sivakumar et al., 2007), analgesic (Sivakumar et al., 2003), anti HIV 
(Tiwari et al., 2000) and antitumor (Ducki et al., 1998) activities.

Herein, we report the synthesis of some novel chalcone analogues using a conventional base catalyzed, Claisen Schmidt condensation reaction and their possible antibacterial activity.

\section{Materials and Methods}

\section{Claisen-Schmidt Condensation}

The most convenient method is the Claisen Schimdt condensation of equimolar quantities of aryl methyl ketone with aryl aldehyde in the presence of alcoholic alkali(Taylor et al., 1967).

The synthesis of chalcone compounds incorporating with hetero cycles became great importance in medicinal chemistry(Padhy et al., 2003; Nakum and Shah, 2002). The hetero atoms in ther structure such as $(\mathrm{S}, \mathrm{N}, \mathrm{O})$ explain variety applications in the biological engineering and in other field of their specific structures(Nagham, 2013).

\section{Experimental}

Melting points of the compounds were determined in open capillary tubes and are uncorrected, IR Spectra were recorded on Shimadzu FT-IR Spectrometer using potassium bromide pellets, ${ }^{1} \mathrm{H}$ NMR was determined on a Bruker Avance II 400 Spectrometer against TMS as internal standard. Mass spectra were recorded on waters Micromass Q-T of Micro spectrometry.

Method for the Synthesis of Novel Chalcones

A mixture of substituted acetophenone (1 mmol $)$, substituted aldehyde $(1 \mathrm{mmol})$ and
$\mathrm{KOH}$ ( 2 mmol, in minium $\mathrm{H} 2 \mathrm{O}$ ) were taken in ethanol and stirred at $50-60^{\circ} \mathrm{C}$ temperature for one hour. The completion of reaction was monitored by TLC. The products were isolated by acidification of the cool diluted acid solution and obtained solid product was filtered and washed with water and recrystallize by ethanol to get pure product.

\section{Results and Discussion}

The synthesis of the newly chalcones were accomplished according to the ClaisenSchmidt condensation of ortho hydroxy ketones with several aromatic aldehyde under microwave irradiation, as indicated to Scheme1. The corresponding reactions proceeded smoothly and in good to excellent yields (70-95 \%). The newly synthesized chalcones were characterized by their chemical, physical and spectral analysis data and are further subjected to antimicrobial studies which exhibit moderate to good activity.

\section{Spectral Analysis of the Compounds}

The newly compounds were done by spectral analysis (IR, ${ }^{1} \mathrm{H}$ NMR,MASS) and the results are shown below :

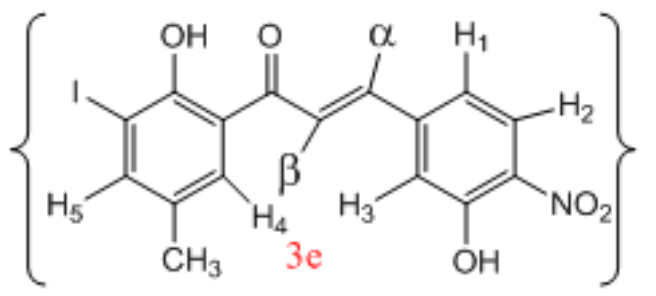

3e- (E)-1-(2-hydroxy-3-iodo-5-methyl phenyl)-3-(3-hydroxy-4-nitrophenyl)prop -2-en-1-one

Compound 3e:- FTIR (KBr, $\left.\mathrm{cm}^{-1}\right)$ : $1617(\mathrm{C}=\mathrm{O}), 1581(\mathrm{C}=\mathrm{C}), 1439(\mathrm{C}-\mathrm{C}$ Aromatic str),1313(N-O sym.stretch). 
${ }^{1}$ HNMR:- 2.33(s, 3H, $\left.\mathrm{CH}_{3}\right), 7.21\left(\mathrm{~d}, 1 \mathrm{H}, \mathrm{H}_{1}\right)$, $7.58\left(\mathrm{~s}, \quad 1 \mathrm{H}, \quad \mathrm{H}_{3}\right), \quad 7.77\left(\mathrm{~d}, \quad 1 \mathrm{H}, \mathrm{H}_{4}\right)$, 7.62(s,1H,OH), 7.81(d, $1 \mathrm{H}, \quad \mathrm{H \alpha}, \mathrm{J}=15 \mathrm{~Hz})$, 7.97(d, $\left.1 \mathrm{H}, \mathrm{H}_{2}\right), 8.12(\mathrm{~d}, 1 \mathrm{H}, \mathrm{H} \beta, \mathrm{J}=15 \mathrm{~Hz})$, $8.21\left(\mathrm{~s}, 1 \mathrm{H}, \mathrm{H}_{5}\right), 13.40(\mathrm{~s}, 1 \mathrm{H}, \mathrm{OH}$ ortho)

M.S. $(\mathbf{m} / \mathbf{z}):(M)=424(M-1)$.

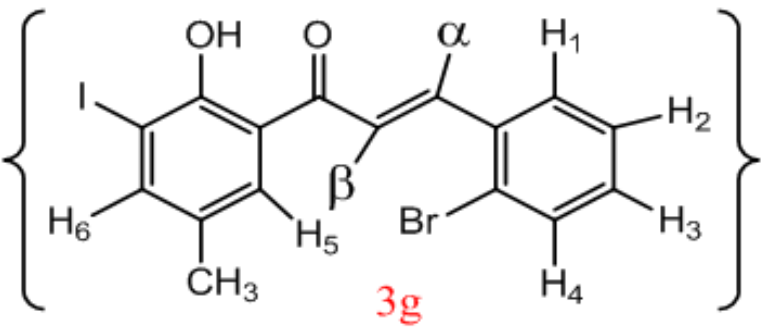

3g- (E)-3-(2-bromophenyl)-1-(2-hydroxy3-iodo-5-methylphenyl)prop-2-en-1-one

Compound 3g:- FTIR ( $\left.\mathrm{KBr}, \mathrm{cm}^{-1}\right)$ : 1631 $(\mathrm{C}=\mathrm{O}), 1565(\mathrm{C}=\mathrm{C}), 1435(\mathrm{C}-\mathrm{C}$ Aromatic str),660(C-Br).

${ }^{1}$ HNMR:- 2.30(s, 3H, $\left.\mathrm{CH}_{3}\right), 7.37\left(\mathrm{dd}, 1 \mathrm{H}, \mathrm{H}_{2}\right)$, 7.46(d, $1 \mathrm{H}, \mathrm{H} \alpha, \mathrm{J}=15 \mathrm{~Hz}), 7.62\left(\mathrm{~d}, 1 \mathrm{H}, \mathrm{H}_{1}\right)$, 7.67(dd, $\left.1 \mathrm{H}, \mathrm{H}_{3}\right), 2.87\left(\mathrm{~d}, 1 \mathrm{H}, \mathrm{H}_{4}\right), 8.06(\mathrm{~d}$, $\left.1 \mathrm{H}, \mathrm{H}_{5}\right), 8.14(\mathrm{~d}, 1 \mathrm{H}, \mathrm{H} \beta, \mathrm{J}=15 \mathrm{~Hz}), 8.17(\mathrm{~d}$, $\left.1 \mathrm{H}, \mathrm{H}_{6}\right), 13.40(\mathrm{~s}, 1 \mathrm{H}, \mathrm{OH})$.

M.S. $(\mathbf{m} / \mathbf{z}):(M)=443(M+), \quad 442(M-1)$, 444(M+1).<smiles>B/C(C(=O)c1c(C)c(C)c(C)c(I)c1O)=C(/Cl)c1c(C)c(CCCCCCC)c(C)c(C)c1Cl</smiles>

3h- (E)-3-(2-chlorophenyl)-1-(2-hydroxy3-iodo-5-methylphenyl)prop-2-en-1-one

Compound 3h:- FTIR (KBr, $\left.\mathbf{~ c m}^{-1}\right)$ : $1631(\mathrm{C}=\mathrm{O}), 1564(\mathrm{C}=\mathrm{C}), 1435(\mathrm{C}-\mathrm{C}$ Aromatic str), $729(\mathrm{C}-\mathrm{Cl})$.
${ }^{1}$ HNMR:- 2.31(s, 3H, $\left.\mathrm{CH}_{3}\right), 7.37\left(\mathrm{dd}, 1 \mathrm{H}, \mathrm{H}_{2}\right)$, $7.45(\mathrm{~d}, 1 \mathrm{H}, \mathrm{H \alpha}, \mathrm{J}=15 \mathrm{~Hz}), 7.53\left(\mathrm{~d}, 1 \mathrm{H}, \mathrm{H}_{1}\right)$, $7.65\left(\mathrm{dd}, 1 \mathrm{H}, \mathrm{H}_{3}\right), 7.88\left(\mathrm{~d}, 1 \mathrm{H}, \mathrm{H}_{4}\right), 8.09(\mathrm{~d}$, $1 \mathrm{H}, \mathrm{H} \beta, \mathrm{J}=15 \mathrm{~Hz}), 8.19\left(\mathrm{~d}, 1 \mathrm{H}, \mathrm{H}_{5}\right), 8.23(\mathrm{~d}$, $\left.1 \mathrm{H}, \mathrm{H}_{6}\right), 13.38(\mathrm{~s}, 1 \mathrm{H}, \mathrm{OH})$.

M.S. $(\mathbf{m} / \mathbf{z})$ : $(\mathrm{M})=397(\mathrm{M}-1), 399(\mathrm{M}+1)$.

\section{Antimicrobial Activity}

Antimicrobial screening was done using disc diffusion method (Afaf et al., 2000) at a concentration of $100 \mu \mathrm{g} / \mathrm{ml}$.

Procedure:- The test was performed according to the disk diffusion method 26 adopted with some modification for the prepared compound using Penciline and streptomycin as references. The prepared compounds were tested against one strain of Gram +ve bacteria, Gram -ve bactria, fungi. Whatman filter paper disk of $5 \mathrm{~mm}$ diameter were sterilized by autoclaving for $15 \mathrm{~min}$ at $1210 \mathrm{c}$. The sterile disk were impregnated with different compounds $(600 \mathrm{gm} /$ disk$)$. Agar plates were surface inoculated uniformly from the both culture of the tested microorganism. The disk were placed on the medium suitably spaced apart on the plate were incubated at $500 \mathrm{C}$ for $1 \mathrm{hr}$ to permit good diffusion and then transferred to an incubator at 370C. for $24 \mathrm{hr}$ for bacteria and $280 \mathrm{C}$ for $72 \mathrm{hrs}$ for fungi.

The compounds were evaluated for antibacterial activity against Staphylococcus aureus gr +ve, Escherichia coli gr -ve Bacillus subtilis $\mathrm{gr}+\mathrm{ve}$, Salmonela typhi gr $-\mathrm{ve}$, and antifungal activity against Aspergillus oryzoe, Aspergillus niger,. DMSO was used as solvent control. The results of antimicrobial data are summarized in table 3. The compounds show the moderate to good activity against bacteria and fungui. 
Table.1 Physical Data of Synthesized Chalcones

\begin{tabular}{|c|c|c|c|c|c|}
\hline Comp.no & Product & $\begin{array}{c}\text { Mol. } \\
\text { Formula }\end{array}$ & $\begin{array}{c}\text { Yield } \\
\text { \% }\end{array}$ & M.P. $\left({ }^{\circ} \mathbf{C}\right)$ & Solubility \\
\hline $3 \mathrm{a}$ & $3 \mathrm{a}$ & $\mathrm{C}_{16} \mathrm{H}_{13} \mathrm{O}_{3} \mathrm{I}$ & 85 & $104-106$ & DMF \\
\hline $3 \mathrm{~b}$ & $3 \mathrm{~b}$ & $\mathrm{C}_{16} \mathrm{H}_{12} \mathrm{O}_{2} \mathrm{IBr}$ & 75 & $154-156$ & DMF \\
\hline $3 \mathrm{c}$ & $3 \mathrm{c}$ & $\mathrm{C}_{16} \mathrm{H}_{13} \mathrm{O}_{4} \mathrm{NI}$ & 70 & $186-190$ & DMF \\
\hline $3 \mathrm{~d}$ & $3 \mathrm{~d}$ & $\mathrm{C}_{15} \mathrm{H}_{12} \mathrm{O}_{2} \mathrm{IN}$ & 75 & $86-90$ & DMF \\
\hline $3 \mathrm{e}$ & $3 \mathrm{e}$ & $\mathrm{C}_{16} \mathrm{H}_{12} \mathrm{O}_{5} \mathrm{~N}$ I & 80 & $194-196$ & DMF \\
\hline $3 \mathrm{f}$ & $3 \mathrm{f}$ & $\mathrm{C}_{15} \mathrm{H}_{13} \mathrm{O}_{2} \mathrm{IS}$ & 85 & $106-108$ & DMF \\
\hline $3 \mathrm{~g}$ & $3 \mathrm{~g}$ & $\mathrm{C}_{16} \mathrm{H}_{12} \mathrm{O}_{2} \mathrm{BrI}$ & 90 & 130 & DMF \\
\hline $3 \mathrm{~h}$ & $3 \mathrm{~h}$ & $\mathrm{C}_{16} \mathrm{H}_{12} \mathrm{O}_{2} \mathrm{ClI}$ & 80 & $118-120$ & DMF \\
\hline $3 \mathrm{i}$ & $3 \mathrm{i}$ & $\mathrm{C}_{15} \mathrm{H}_{12} \mathrm{O}_{2} \mathrm{NI}$ & 75 & $94-98$ & DMF \\
\hline $3 \mathrm{j}$ & $3 \mathrm{j}$ & $\mathrm{C}_{18} \mathrm{H}_{14} \mathrm{O}_{2} \mathrm{NI}$ & 95 & $110-112$ & DMF \\
\hline
\end{tabular}

Table.2 Antimicrobial Activity of Synthesized Compounds

\begin{tabular}{|c|c|c|c|c|c|c|}
\hline compounds & \multicolumn{2}{|c|}{ Gram positive bacterias } & Gram negative bacterias & \multicolumn{2}{c|}{ Fungus } \\
\hline & Staph aureus & $\begin{array}{c}\text { Bacillus } \\
\text { subtilis }\end{array}$ & $\begin{array}{c}\text { Escherichia } \\
\text { coli }\end{array}$ & S. typhi & $\begin{array}{c}\text { Aspergillus } \\
\text { oryzoe }\end{array}$ & $\begin{array}{c}\text { Aspergillus } \\
\text { niger }\end{array}$ \\
\hline $3 \mathrm{a}$ & - & - & + & - & - & - \\
\hline $3 \mathrm{~b}$ & + & - & - & - & - & - \\
\hline $3 \mathrm{c}$ & ++ & + & - & - & + & ++ \\
\hline $3 \mathrm{~d}$ & + & - & & & - & ++ \\
\hline $3 \mathrm{e}$ & + & + & - & - & - & + \\
\hline $3 \mathrm{f}$ & - & - & & & - & + \\
\hline $3 \mathrm{~g}$ & + & - & - & - & - & - \\
\hline $3 \mathrm{~h}$ & + & - & - & - & - & - \\
\hline $3 \mathrm{i}$ & ++ & + & - & - & + & + \\
\hline $3 \mathrm{j}$ & + & + & + & + & + & + \\
\hline DMSO & - & - & - & - & - & - \\
\hline Penciline 1 & ++ & - & + & - & $\mathrm{x}$ & $\mathrm{x}$ \\
\hline Streptomycin 2 & ++ & ++ & ++ & ++ & $\mathrm{x}$ & $\mathrm{x}$ \\
\hline Greseofulvin & $\mathrm{x}$ & $\mathrm{x}$ & $\mathrm{x}$ & $\mathrm{x}$ & - & - \\
\hline
\end{tabular}

$++=$ Clear Zone of Inhibition

$+=$ Minimum Zone of Inhibition

- = No Effect

$\mathrm{X}=$ Not applicable

Standerd 1 Penciline +, Standerd 2 Streptomycin ++ (bacteria). Greseofulvin (fungus) 
Int.J.Curr.Microbiol.App.Sci (2016) 5(3): 785-791

Scheme.1 Synthesis of Chalcones

Comp.no


Reagents.(a) aq. $\mathrm{KOH}$, Alcohol<smiles>[R]C=CC(=O)c1cccc([R])c1</smiles>

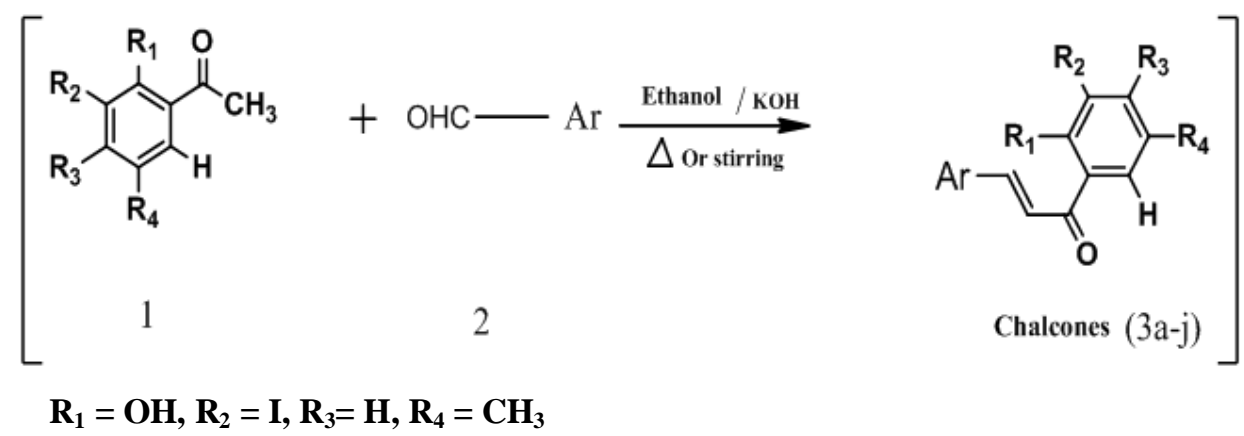

In conclusion, here we have reported some novel chalcones using ortho hydroxyl acetophenone with several aromatic aldehydes with high yield. The newly synthesized chalcones were confirmed by spectral analysis and further evaluated for their antimicrobial activity. The antibacterial activity revealed that of the compounds showed moderate to good activity against the pathogens used.

\section{References}

Afaf, H., El-masry, H.H., Fahmy, Ali, S.H., Abdelwahed. 2000. Molecules, 5(12): 1429-1438.

Ballesteros, J.F., Sanz, M.J., Ubeda, A., Miranda, M.A., Iborra, S., Paya, M., Alcaraz, M. 199. J. Med. Chem., 38: 2794.

Bergman, E.D., Ginsibm, L., Pappo, R. 1959. Org. React., 10: 179.

Chikhalia, K.H., Patel, M.J., Vashi, D.B. 2008. Design, synthesis and evaluation of novel quinolinylchalcones as antibacterial agents. ARKIVOC, 9: 189197.

Ducki, S., Forrest, R., Hadfield, J.A., Kendall, A., Lawrence, N.J., Mc-Gown, A.T., Rennison, D. 1998. Bio. org. Med. Chem., 8: 1051.

Go, M.L., Wu, X., Liu, X.L. 2005. Chalcones: An update on cytotoxic and chemoprotective properties. Current Med. Chem., 12(4): 483-499.

Go, M.L., Wu, X., Liu, X.L. 2005. Curr. Med. Chem., 12: 483.

Kotra, V., Ganapaty, S., Adapa, S.R. 2010. Synthesis of a new series of quinolinylchalcones as anticancer and anti-inflammatory agents. Indian $J$. Chem., 49B(8): 1109-1116.

Liu, U.M., Wilairat, P., Croft, S.L., Tan, A.L., Go, M. 2003. Bioorg. Med.Chem., 11: 2729 .

Mukerjee, V.K., Prased, A.K., Raj, A.G., Brakhe, M.E., Olsen, C.E., Jain, S.C., 
Parmer, V.P. 2001. Bioorg. Med. Chem., 9: 337.

Nagham, M.A. 2013. J. Chem \& Chemi. Sci., 3(2): 70-78.

Nakum, K.H., Shah, V.H. 2002. Indian J. Het. Chem., 12(1): 37.

Padhy, A.K., Bardham, M., Danda, C.S. 2003. Indian J. Chem., 42B(4): 910.

Sandler, S., Karo, W. 1972. In Organic Functional Group Preparations. 3: 372.

Satyanarayana, M., Tiwari, P., Tripathi, B.K., Srivastava, A.K., Pratap, R. 2004. Synthesis and antihyperglycemic activity of chalcone based aryloxypropanolamines. Bioorg. Med. Chem., 12(5): 883-889.

Sivakumar, P.M., Geetha Babu, S.K., Mukesh, D. 2007. Chem. Pharm. Bull., 55: 44.

Straub, T.S. 1995. Tetrahedron Lett., 36: 663.

Taylor, E.C., Morrison, R.W., 1967. J. Org. Chem., 32: 2379(Claisen Schmidt condensation)

Tiwari, N., Dwivedi, B., Nizamuddin, K.F., Nakanshi, Y., Lee, K.H. 2000. Bio. org.Med. Chem., 10: 699.

Viana, G.S., Bandeira, M.A., Mantos, F. 2003. J. Phytomedicine, 10: 189.

\section{How to cite this article:}

Abdulkareem Ali Hussien, Bushra Ahmed Kateb, Shekh Saddam Husen and Kulkarni, P. A. 2016. Synthesis, Characterization and Antimicrobial Screening of Novel Ortho Hydroxy Chalcones. Int.J.Curr.Microbiol.App.Sci. 5(3): 785-791.

doi: http://dx.doi.org/10.20546/ijcmas.2016.503.091 